\title{
Density, heterogeneity and deformability of red cells as markers of clinical severity in hereditary spherocytosis
}

Haematologica 2020

Volume 105(2):338-347

\section{Correspondence: \\ RICHARD VAN WIJK \\ r.vanWijk@umcutrecht.nl \\ Received: April 3, 2018. \\ Accepted: May 28, 2019. \\ Pre-published: May 30, 2019.}

doi:10.3324/haematol.2018.188151

Check the online version for the most updated information on this article, online supplements, and information on authorship \& disclosures: www.haematologica.org/content/105/2/338

\section{(C)2020 Ferrata Storti Foundation}

Material published in Haematologica is covered by copyright. All rights are reserved to the Ferrata Storti Foundation. Use of published material is allowed under the following terms and conditions:

https://creativecommons.org/licenses/by-nc/4.0/legalcode. Copies of published material are allowed for personal or internal use. Sharing published material for non-commercial purposes is subject to the following conditions:

https://creativecommons.org/licenses/by-nc/4.0/legalcode, sect. 3. Reproducing and sharing published material for commercial purposes is not allowed without permission in writing from the publisher.

\author{
Rick Huisjes, ${ }^{1}$ Asya Makhro, ${ }^{2}$ Esther Llaudet-Planas, ${ }^{3}$ Laura Hertz, ${ }^{4}$ Polina \\ Petkova-Kirova, ${ }^{4}$ Liesbeth P. Verhagen, ${ }^{1}$ Silvia Pignatelli, ${ }^{1}$ Minke A.E. Rab, ${ }^{1}$ \\ Raymond M. Schiffelers, ${ }^{1}$ Elena Seiler, ${ }^{2}$ Wouter W. van Solinge, ${ }^{1}$ Joan-LLuis \\ Vives Corrons, ${ }^{3}$ Lars Kaestner, ${ }^{4,5}$ Maria Mañú-Pereira, ${ }^{6}$ Anna Bogdanova ${ }^{2}$ and \\ Richard van Wijk ${ }^{1}$
}

${ }^{1}$ Department of Clinical Chemistry and Hematology, University Medical Center Utrecht, Utrecht University, Utrecht, the Netherlands; ${ }^{2}$ Red Blood Cell Research Group, Institute of Veterinary Physiology, Vetsuisse Faculty and the Zurich Center for Integrative Human Physiology (ZIHP), University of Zurich, Zurich, Switzerland; ${ }^{3}$ Red Blood Cell Defects and Hematopoietic Disorders Unit, Josep Carreras Leukemia Research Institute, Badalona, Barcelona, Spain; ${ }^{4}$ Theoretical Medicine and Biosciences, Medical Faculty, Saarland University, Homburg/Saar, Germany; ${ }^{5}$ Experimental Physics, Saarland University, Saarbruecken, Germany and 'Rare Anemia Research Unit. Vall d'Hebron Research Institution, University Hospital Vall d'Hebron, Barcelona, Spain

\section{ABSTRACT}

$\mathrm{H}$ ereditary spherocytosis (HS) originates from defective anchoring of the cytoskeletal network to the transmembrane protein complexes of the red blood cell (RBC). Red cells in HS are characterized by membrane instability and reduced deformability and there is marked heterogeneity in disease severity among patients. To unravel this variability in disease severity, we analyzed blood samples from $21 \mathrm{HS}$ patients with defects in ankyrin, band $3, \alpha$-spectrin or $\beta$-spectrin using red cell indices, eosin-5maleimide binding, microscopy, the osmotic fragility test, Percoll density gradients, vesiculation and ektacytometry to assess cell membrane stability, cellular density and deformability. Reticulocyte counts, CD71 abundance, band $4.1 \mathrm{a}: \mathrm{b}$ ratio, and glycated hemoglobin were used as markers of RBC turnover. We observed that patients with moderate/severe spherocytosis have short-living erythrocytes of low density and abnormally high intercellular heterogeneity. These cells show a prominent decrease in membrane stability and deformability and, as a consequence, are quickly removed from the circulation by the spleen. In contrast, in mild spherocytosis less pronounced reduction in deformability results in prolonged RBC lifespan and, hence, cells are subject to progressive loss of membrane. RBC from patients with mild spherocytosis thus become denser before they are taken up by the spleen. Based on our findings, we conclude that RBC membrane loss, cellular heterogeneity and density are strong markers of clinical severity in spherocytosis.

\section{Introduction}

Hereditary spherocytosis (HS) is the most common form of chronic hereditary hemolytic anemia in the Caucasian population, with an estimated prevalence of 1:2000 - 1:5000. $\cdot^{1-3}$ HS usually originates from mutations in ANK1 (ankyrin), SLC4A1 (band 3), SPTA1 ( $\alpha$-spectrin), SPTB ( $\beta$-spectrin) or EPB42 (protein 4.2). ${ }^{1}$ Anemia in HS may require transfusion(s) and in severe cases splenectomy. A characteristic feature of $\mathrm{HS}$ is red blood cell (RBC) membrane instability, which leads to membrane loss and formation of dense cells with reduced RBC deformability. ${ }^{46}$ Increased RBC density is an important feature of $\mathrm{HS}^{7}$ and is reflected, for example, by increased mean corpuscular hemoglobin concentration (MCHC).,9,

$\mathrm{HS}$ is a very heterogeneous RBC disorder, resulting from a wide range of molecular defects and characterized by a high degree of heterogeneity in RBC properties and disease severity. ${ }^{8-12}$ In fact, considerable differences in disease severity are reported even between HS patients with identical mutations. It therefore seems rea- 
sonable to assume that the heterogeneity in disease severity is not only a reflection of particular genotypes but is also affected by other factors that control RBC properties.

Healthy RBC become increasingly dense during their lifespan, ${ }^{13,14}$ but this process is accelerated in HS. ${ }^{1}$ Shedding of essentially hemoglobin-free vesicles results in an increase in $\mathrm{MCHC}$ and a corresponding increase in $\mathrm{RBC}$ density and intracellular viscosity ${ }^{13,15}$ Electrogenic potassium leakage also contributes to RBC dehydration in HS patients and is not compensated by an accumulation of $\mathrm{Na}^{+}{ }^{16,17}$ As a result, the intracellular $\mathrm{K}^{+}$concentration in the $\mathrm{RBC}$ of HS patients is approximately $13 \mathrm{mmol} / \mathrm{L}$ lower than that in the cells of healthy subjects, ${ }^{16}$ which results in net ion and water loss. Compensatory activation of $\mathrm{Na}, \mathrm{K}$ ATPase in the RBC of patients is insufficient to prevent the loss of $\mathrm{K}^{+}$and dissipation of $\mathrm{K}^{+} / \mathrm{Na}^{+}$gradients. The function of other electroneutral ion transporters (KCC, NKCC, $\mathrm{Na} / \mathrm{Li}$ exchanger) in $\mathrm{RBC}$ of $\mathrm{HS}$ patients was reported to be indistinguishable from that of cells of healthy controls. ${ }^{16,18}$

In this study, we investigated a unique and genetically well-diagnosed group of HS patients in whom we performed an in-depth analysis of RBC properties, such as membrane instability, cellular density, cellular heterogeneity, vesiculation, turnover and lifespan. The data obtained were then correlated to clinical manifestations of HS in both non-splenectomized and splenectomized patients, in order to identify markers of disease severity. Our results indicate that clinical severity in HS cannot be solely attributed to the protein harboring the mutation, but rather to the stability of the whole cytoskeletal network. RBC density, heterogeneity and deformability were identified as potential markers of severity. We found that the presence of dense RBC is strongly associated with milder manifestations of HS. We hypothesize that unstable RBC from patients with clinically more severe disease are removed from the circulation before they acquire the features of senescence.

\section{Methods}

\section{Subjects}

Patients previously diagnosed with HS were enrolled in the CoMMiTMenT-study (http://www.rare-anaemia.eu). This study was approved by the Medical Ethical Research Board of the University Medical Center Utrecht, the Netherlands, under reference code $15 / 426 \mathrm{M}$ and by the Ethical Committee of Clinical Investigations of Hospital Clinic, Spain, (IDIBAPS) under reference code 2013/8436.

\section{Hemocytometry analysis}

Hemocytometry parameters were analyzed on an Abbott Sapphire cell analyzer (Abbott Diagnostics Division, Santa Clara, CA, USA) and ADVIA 2120 (Hematology System, Siemens Healthcare Diagnostics, Forchheim, Germany).

\section{Capillary-based measurements of mean corpuscular volume and mean corpuscular hemoglobin concentration}

Triplicate heparinized blood samples were put in capillaries and centrifuged for $5 \mathrm{~min}$ at 12,000 rpm (Hematocrit 20, Hettich Zentrifugen). Mean corpuscular volume (MCV) was calculated using the formula $\mathrm{MCV}=$ hematocrit/RBC number. $\mathrm{MCHC}$ was calculated using the formula $\mathrm{MCHC}=$ hemoglobin/hematocrit.
Separation on a Percoll density gradient and determination of intracellular potassium levels

Intact blood samples were layered over a 90\% isotonic Percoll solution containing plasma-like components as described elsewhere. ${ }^{19}$ Briefly, Percoll density gradient and RBC separation were performed during centrifugation at 50,000 g for $15 \mathrm{~min}$. (Sorvall RC 5C plus, rotor SM-24). Intracellular potassium was measured using an Instrumentation Laboratory IL943 Flame Photometer, as described by Jokinen et al. ${ }^{20}$

\section{Osmotic gradient ektacytometry, the osmotic fragility test and eosin-5-maleimide binding}

Osmotic gradient ektacytometry measurements of RBC from healthy controls and HS patients were obtained using the Osmoscan module on a Lorrca MaxSis (Mechatronics, The Zwaag, the Netherlands) as described elsewhere., ${ }^{5,21}$ The osmotic fragility test was carried out as previously described by Parpart et al. $^{22}$ and eosin-5-maleimide (EMA) binding was determined according to previously published protocols. ${ }^{12,23}$

Red blood cell production, heterogeneity, vesiculation and turnover rate markers

$\mathrm{RBC}$ were stained with anti-CD71 and isotype controls and were subsequently measured using a BD FACS Gallios. ${ }^{24}$ Measuring glycated hemoglobin (HbA1c) is an established way to acquire information about $\mathrm{RBC}$ clearance and $\mathrm{RBC}$ age in research on hemolytic anemias. ${ }^{15,25} \mathrm{HbA} 1 \mathrm{c}$ levels were measured using a Menarini/ARKRAY HA-8180V. The band 4.1a:b ratio was detected in RBC membrane lysates after protein separation by inverse sodium dodecylsulfate polyacrylamide gel electrophoresis (SDSPAGE) (15-7.5\%) and visualization of protein bands using Coomassie blue staining. RBC projected area and its heterogeneity were evaluated by microscopy as described elsewhere. ${ }^{26,27} \mathrm{RBC}$ vesicles were identified in plasma preserved with citrate-phosphate-dextrose-adenine ${ }^{19}$ by staining them with mouse antihuman CD235a-APC and measured using a Beckman Coulter CytoFLEX flow cytometer (Online Supplementary Figure S1).

\section{Statistical analysis and phenotype correlations}

One-way analysis of variance with post-hoc correction (Tukey test) was used to compare sample means and the Fisher exact test was applied to determine whether clinical severity was proportionally distributed along the different genotypes. In non-splenectomized patients, clinical severity was assessed based on: (i) hemoglobin concentration and (ii) reticulocyte count, as previously defined by Bolton-Maggs et al. ${ }^{11}$ (i.e., mild and moderate/severe). To prevent any confounding by splenectomized patients, phenotype correlations were only carried out for unsplenectomized HS patients.

\section{Results}

\section{Baseline characteristics and red cell features of patients with hereditary spherocytosis}

Twenty-one patients with HS were included in this study and categorized according to clinical severity (Table 1). ${ }^{11}$ HS was confirmed by targeted next-generation sequencing of the seven genes most commonly mutated in $\mathrm{HS}^{28}$. Splenectomy or a moderate/severe expression of disease was statistically overrepresented in patients with mutations in ANK1 and SPTB $(P<0.05)$. Therefore, the phenotypic expression of HS due to ANK1 and SPTB mutations appears to be more severe than that of HS due to SLC4A1 and/or SPTA1 defects. Decreased EMA stain- 
ing, reflecting band 3 protein loss, was seen in all patients. Patients with SPTA1 mutations tended to have greater EMA staining (Figure 1A), although the number of patients was too low to draw firm conclusions. The maximal deformability of $\mathrm{RBC}$, reflected by a decrease in maximal elongation index $\left(\mathrm{EI}_{\max }\right)$ as determined by the Osmoscan, was decreased in all HS patients compared to that in healthy controls. This decrease was more pronounced in patients with ANK1 and SPTB mutations than in patients with mutations in SPTA or SCL4A1 (Figure 1B). On the other hand, cells from patients with SLC4A1 mutations tended to be more dehydrated as their $\mathrm{O}_{\text {hyper }}$ values (hypertonic osmolarity at $50 \%$ of $\mathrm{EI}_{\max }$ ) were lower than those in both control blood samples and patients with SPTA1 mutations (Figure 1C). The latter patients also showed the least pronounced loss of surface area-tovolume ratio, reflected by a normal $\mathrm{O}_{\min }$ (hypotonic osmolarity where EI is minimal) (Figure 1D) on the Osmoscan and normal results in the osmotic fragility test
(50\% lysis point) (Figure 1E). Membrane stability was compromised in all other HS patients.

As expected, RBC turnover was increased in all patients: reticulocyte counts were high (Table 1) and the band $4.1 \mathrm{a}: \mathrm{b}$ ratio, a marker of aging, was lower in all patients than in healthy controls (Table 1).

The heterogeneity of the $\mathrm{RBC}$, reflected by the red blood cell distribution width (RDW), was greater in patients with HS than in healthy controls (Figure 1F) as was the MCHC (Figure 1G). Intracellular $\mathrm{K}^{+}$content was reduced in all patients but tended to be higher in HS patients with SPTA1 mutations (Figure $1 \mathrm{H}$ ).

An increase in heterogeneity in cell projected areas (mean projected area distribution width, MPA DW) (Figure 2A) and a decrease in absolute mean values in projected area (MPA) (Figure 2B) were seen in all HS patients compared to those in controls. Patients with SLC4A1 and SPTA1 mutations had cellular projected areas more similar to those of the healthy control group (Figure $2 \mathrm{~A}, \mathrm{~B}$ ).

Table 1. Hemocytometry, chemistry parameters, cell-age markers and genotypes of patients with mild or moderate/severe hereditary spherocytosis (HS) and splenectomized HS patients included in this study.

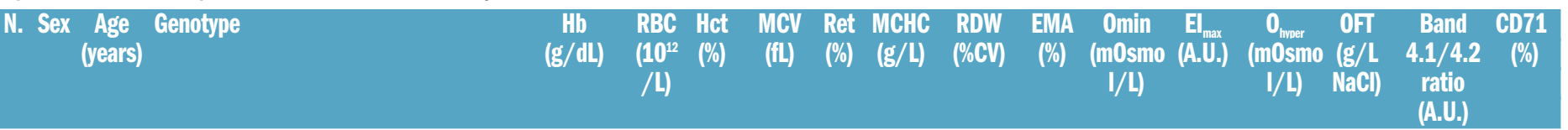

\section{Mild HS ${ }^{11}$}

\begin{tabular}{|c|c|c|c|c|c|c|c|c|c|c|c|c|c|c|c|c|c|}
\hline 1 & $\hat{\delta}$ & 75 & ANK1 c.344T>C p.Leul15Pro & 136 & 4.1 & n.a. & n.a. & 7.2 & n.a. & 16.2 & 85 & 162 & 0.555 & 457 & 7.0 & 0.70 & 0.8 \\
\hline 2 & $\hat{\delta}$ & 46 & SLC4Al c. $1030 \mathrm{C}>\mathrm{T}$ p.Arg344* & 153 & 4.7 & n.a. & n.a. & 3.2 & n.a. & 15.2 & 71 & 163 & 0.563 & 417 & 6.6 & 0.87 & 1.4 \\
\hline 3 & $\hat{\delta}$ & 40 & SLC4AI c.1421C>A p.Ala474Asp & 140 & 4.9 & n.a. & n.a. & 4.0 & n.a. & 12.3 & 93 & 162 & 0.557 & 427 & 5.7 & 0.99 & 1.2 \\
\hline 4 & 우 & 55 & SLC4Al c.2057+1G>A (splicing) & 136 & 4.0 & 36.5 & 91.1 & 7.8 & 372 & 14.0 & 73 & 158 & 0.554 & 405 & 6.4 & 0.82 & 2.7 \\
\hline 5 & 우 & 18 & SLC4AI c.2057+1G>A (splicing) & 131 & 3.7 & 35.7 & 96.9 & 9.4 & 367 & 13.1 & 77 & 166 & 0.569 & 410 & 6.1 & 0.84 & 1.4 \\
\hline 6 & $\hat{\delta}$ & 58 & SLC4A1 c.2348T>A p.Ile783Asn & 132 & 4.1 & 36.5 & 88.4 & 8.9 & 363 & 15.7 & 68 & 169 & 0.548 & 429 & 6.8 & n.a. & 3.5 \\
\hline 7 & $\hat{\delta}$ & 40 & SPTAl c.678G $>$ A p.Glu227fs $+\alpha^{\text {LELIY }}$ & 127 & 4.1 & 35.9 & 87.8 & 5.8 & 355 & 15.0 & 94 & 175 & 0.600 & 453 & 5.6 & 0.84 & 0.9 \\
\hline 8 & $\hat{\delta}$ & 54 & $\begin{array}{l}\text { SPTA1 c. [4339-99C>T; c.4347G > T] } \\
\text { p.[(?; Lys1449Asn)]; c.4339-99C }>\text { T }\end{array}$ & 121 & 3.5 & 34.7 & 98.3 & 3.9 & 348 & 15.3 & 94 & 160 & 0.566 & 458 & 5.7 & n.a. & 0.9 \\
\hline
\end{tabular}

\begin{tabular}{|c|c|c|c|c|c|c|c|c|c|c|c|c|c|c|c|c|c|}
\hline \multicolumn{18}{|c|}{ Moderate/severe $\mathbf{H S}^{11}$} \\
\hline 9 & $\hat{\delta}$ & 4 & $A N K 1$ c.341C $>$ T p.Pro114Leu & 116 & 3.8 & 30.0 & 80.1 & 9.1 & 386 & 14.5 & 72 & 162 & 0.509 & 398 & 6.6 & 0.72 & 2.2 \\
\hline 10 & 우 & 3 & ANK1 c.1943delC p.Ala648fs & 117 & 4.02 & 36.3 & 90.4 & 11.9 & 321 & 21.1 & 59 & 164 & 0.503 & 424 & 6.6 & n.a. & 0.2 \\
\hline 11 & $\hat{\delta}$ & 5 & ANK1 c.2394_2397delCAGT p.Ser799fs & 120 & 4.1 & 32.2 & 78.6 & 18.4 & 372 & 25.4 & 67 & 185 & 0.472 & 462 & 7.8 & n.a. & 7.3 \\
\hline 12 & 우 & 26 & ANK1 c.2559-2A>G (splicing) & 102 & 3.3 & 29.4 & 89,5 & 18.3 & 346 & 24.1 & 84 & 170 & 0.537 & 446 & 6.4 & 0.60 & 5.3 \\
\hline 13 & $\hat{o}$ & 1 & SPTB c.154delC p.Arg52fs & 86 & 3.38 & 30.2 & 89.5 & 11.9 & 284 & 24.7 & 66 & 168 & 0.532 & 456 & 5.7 & n.a. & 4.8 \\
\hline 14 & $\hat{\delta}$ & 3 & SPTB c.2470C>T p.Gln824* & 78 & 2.9 & 23.7 & 81.9 & 10.6 & 328 & 23.8 & 71 & 180 & 0.534 & 475 & 6.7 & n.a. & 2.9 \\
\hline 15 & $\hat{o}$ & 4 & SPTB c.5937+1G>A p.(?) & 84 & 3.09 & 27.7 & 89.7 & 16.7 & 303 & 23.4 & 74 & 173 & 0.541 & 459 & 7.0 & n.a. & 5.5 \\
\hline 16 & 우 & 42 & SPTAl c.2755G >T p.Glu919* $+\alpha^{\text {LEIY }}$ & 113 & 3.5 & 31.7 & 91.8 & 8.2 & 355 & 16.0 & 89 & 178 & 0.567 & 471 & 5.9 & n.a. & 2.1 \\
\hline \multicolumn{18}{|c|}{ Splenectomized HS ${ }^{11}$} \\
\hline 17 & $\hat{\delta}$ & 31 & $A N K 1$ c.341C>T p.Pro114Leu & 152 & 4.9 & 84.7 & 41.9 & 8.4 & 362 & 11.8 & 76 & 179 & 0.533 & 437 & 7.4 & 1.08 & 0.1 \\
\hline 18 & 우 & 46 & ANK1 c.344T>C p.Leul15Pro & 143 & 4.6 & n.a. & n.a. & 2.8 & n.a. & 12.4 & 74 & 158 & 0.590 & 423 & 7.4 & 1.18 & 0.2 \\
\hline 19 & 우 & 84 & SLC4A1 c.2057+1G>A (splicing) & 160 & 4.8 & 121.8 & 45.1 & 12.6 & 354 & 11.0 & 67 & 164 & 0.565 & 417 & 7.1 & 1.07 & 0.2 \\
\hline 20 & $\hat{o}$ & 71 & SPTB c.2136_2137delinsTT & 164 & 4.84 & 100.9 & 48.8 & 2.2 & 337 & 13.2 & 77 & 173 & 0.509 & 445 & 7.3 & 0.99 & 0.3 \\
\hline 21 & $\hat{o}$ & 40 & SPTB c.3449G>A p.Trp1150* & 163 & 4.94 & 92.0 & 45.5 & 12.2 & 359 & 11.9 & 73 & 183 & 0.512 & 440 & 7.8 & 1.22 & 0.3 \\
\hline
\end{tabular}

Clinical severity in non-splenectomized HS patients was assigned according to Bolton-Maggs et al. ${ }^{11}$ on the basis of (i) hemoglobin concentration and (ii) reticulocyte count. Mild HS was defined as hemoglobin levels between 110-150 g/L, moderate HS as hemoglobin levels between 80-120 g/L and severe HS as hemoglobin levels lower than 80 g/L. HS patients with hemoglobin levels between 110 and $120 \mathrm{~g} / \mathrm{L}$ were categorized as having mild or moderate disease on the basis of their reticulocyte levels (i.e., lower or higher than $6 \%$ reticulocytes). Novel mutations are displayed in bold font and the pathogenicity of novel missense variants was predicted with SIFT, PolyPhen-2, and MutationTaster (results not shown). Notation of $\alpha$ LELY represents SPTA1 c. [5572C $>$ G; 6531 $12 \mathrm{C}>\mathrm{T}$ ] p. [(Leu 1858Val);(?)]. N: number; Hb: hemoglobin; RBC: red blood cells; Hct: hematocrit; MCV; mean corpuscular volume; Rct: reticulocytes; MCHC: mean corpuscular hemoglobin concentration; RDW: red cell distribution width; EMA: eosin-5'-maleimide; \%CV: percent coefficient of variation; HS: hereditary spherocytosis; n.a.: not available. 
Patients with HS presented with greater heterogeneity in cell density compared with healthy subjects, showing more subfractions of the $\mathrm{M}$ fraction and a broader distribution width of the $\mathrm{M}$ fraction (Figure 2C-E). These changes were more pronounced in patients with mutations in ANK1 and SPTA1. Furthermore, the M fraction of patients with SLC4A1 mutations was lower than that of healthy controls or other HS patients (Figure 2F, Online Supplementary Figure S2).

In summary, specific changes were observed in parameters associated with membrane stability, stiffness and deformability, as well as RBC heterogeneity in our cohort of HS patients.

\section{Red blood cell markers of severity of hereditary spherocytosis}

As described in Table 1, the severity of HS in nonsplenectomized patients was determined based on the decrease in hemoglobin concentration and increase in reticulocyte count. ${ }^{11}$ In our patients hemoglobin concentration correlated positively with MCHC (Figure 3A). Inverse correlations were observed between hemoglobin and RDW (Figure 3B), and between hemoglobin and reticulocyte count (Figure 3C). Furthermore, inverse correlations were observed between hemoglobin and parameters defining RBC hydration status such as intracellular $\mathrm{K}^{+}$and $\mathrm{O}_{\text {hyper }}$ (Figure 3D, E).

Decreases in RBC lifespan were assessed by a reduction in changes in $\mathrm{HbA1}$ c in non-splectomized patients with severe HS, whereas in patients with mild disease manifestations or in splenectomized patients $\mathrm{HbA1c}$ levels were within the normal range (Figure 4A).

Patients with moderate/severe HS had less deformable $\mathrm{RBC}$, reflected by lower $\mathrm{El}_{\max }$ values, than patients with mild $\mathrm{HS}$ or splenectomized patients (Figure 4B). The RBC of patients with mild HS also tended to be more

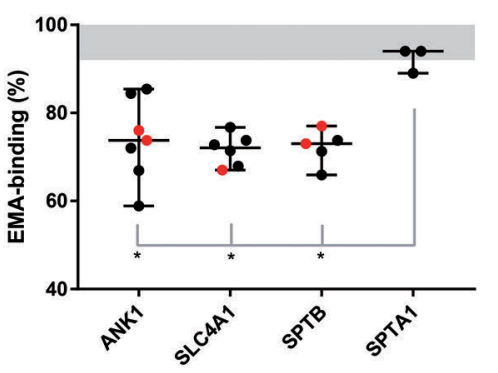

D
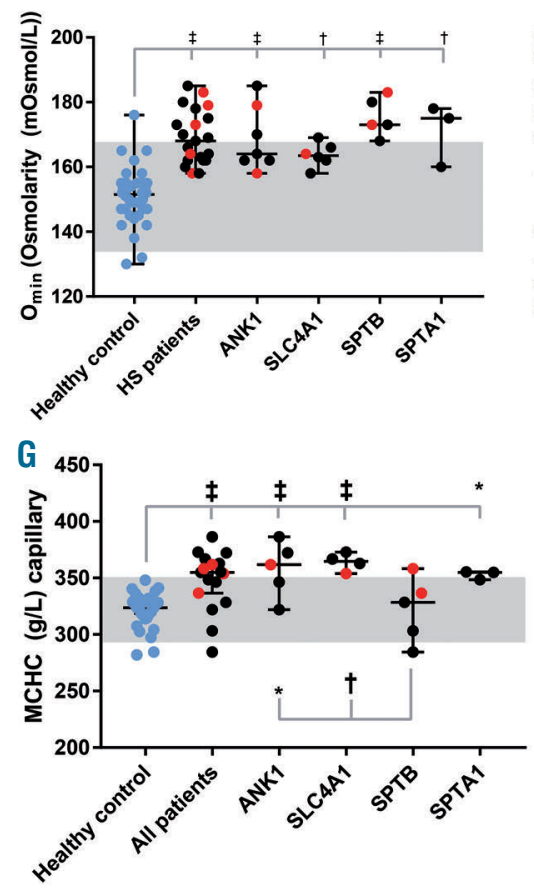

B

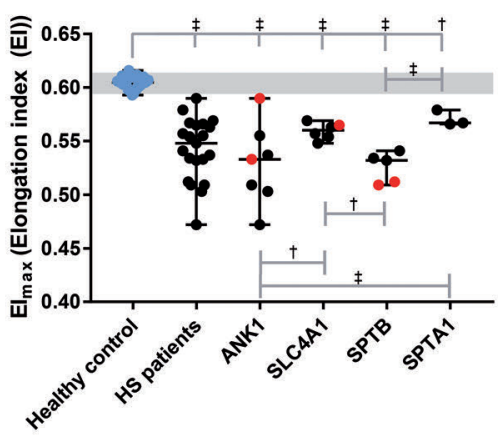

E

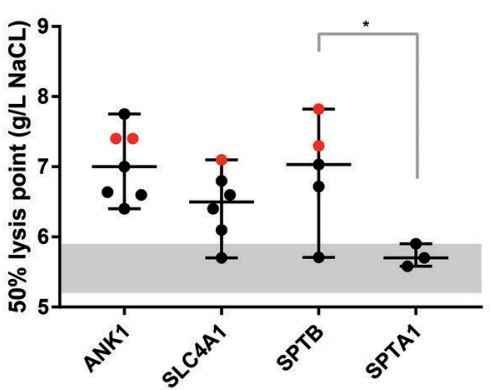

H

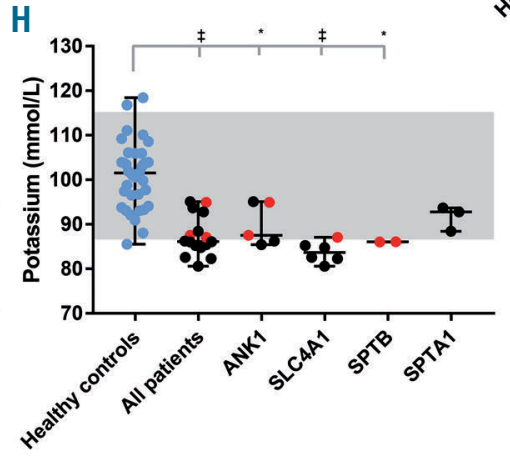

C

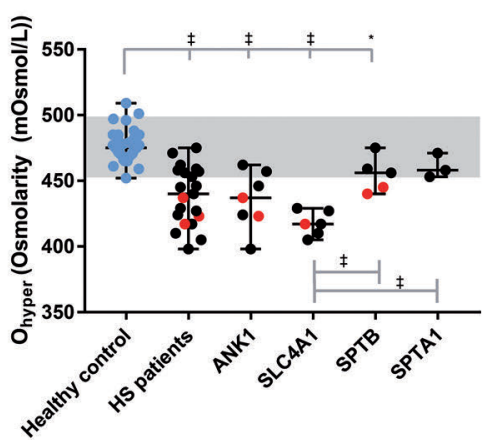

F
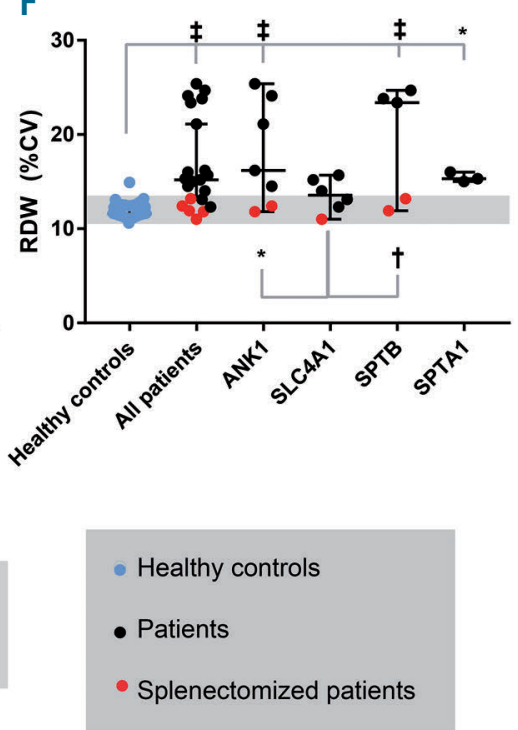

Figure 1. Basic characteristics (median \pm range) of healthy controls and patients with hereditary spherocytosis. Patients with hereditary spherocytosis (HS) were grouped as a whole and according to their affected genes [ANK1 (ankyrin), SLC4A1 (band 3), SPTB ( $\beta$-spectrin) and SPTA1 ( $\alpha$-spectrin)]. Blue circles represent healthy controls, black circles represent unsplenectomized HS patients and red circles represent splenectomized HS patients. The gray range indicates the reference range for healthy controls. (A) Eosin-5'-maleimide (EMA)-binding (\%), (B) maximum deformability (EI max ), (C) hydration state of the red blood cells reflected by $\mathrm{O}_{\text {hyper, }}$, (D) osmotic fragility measured by osmotic gradient ektacytometry and reflected by Omin, (E) $50 \%$ lysis point in the osmotic fragility test, (F) red blood cell distribution width (RDW) (percent coefficient of variation, \%CV), (G) mean corpuscular hemoglobin concentration (MCHC) by capillary measurements ( $\mathrm{g} / \mathrm{L}$ ) (\%CV), (H) intracellular potassium (mmol/L). Significant differences are depicted with horizontal bars, and significance levels are noted: ${ }^{\star} P \leq 0.05,{ }^{\dagger} P \leq 0.01$ or ${ }^{\ddagger} P \leq 0.001$. 
dehydrated, based on the $\mathrm{O}_{\text {hyper }}$ measurements (Figure 4C). In line with this, patients with mild HS showed higher MCHC values than moderately/severely affected patients (Figure 4D) and had a higher density of the M fraction, based on this latter's position within the Percoll gradient (Figure 4E, Online Supplementary Figure S2).

Based on reduced MPA, membrane loss was more pronounced in patients with moderate/severe HS (Figure $4 \mathrm{~F})$. In line with this, the number of $\mathrm{RBC}$ vesicles detected in the plasma of patients with moderate/severe HS was higher than that in patients with mild HS (Figure $4 G)$. However, no difference in EMA staining was observed between patients with mild or moderate/severe HS (Figure 4C). Intercellular heterogeneity (RDW and MPA DW) was increased in patients with moderate/severe HS compared to those with mild HS and healthy controls (Figure 4I, J).

In summary, the RBC of patients with more severe expression of the disease had a reduced lifespan and less stable membrane. Their cells were smaller and more heterogeneous in size and density. Strikingly, patients with mild HS had denser RBC with higher MCHC (Figure 4D, E).

\section{Effect of splenectomy on red blood cell markers of disease severity}

Performed in patients with moderate/severe HS, splenectomy results in an increase in hemoglobin levels and erythrocyte counts (data not shown). In our cohort splenectomy was also associated with a decrease in RDW (Figure 5A) and normalization of RBC morphology (Online Supplementary Figures S3 and S4). The survival of RBC from splenectomized $\mathrm{HS}$ patients, as assessed by $\mathrm{HbA1c}$ content or band $4.1 \mathrm{a}: \mathrm{b}$ ratio, was found to be increased (Figure 5B, C).

Several parameters remained unaffected by splenectomy. Splenectomy did not alter MCHC (Figure D) or intracellular $\mathrm{K}^{+}$levels (Figure $5 \mathrm{E}$ ) and did not correct band 3 loss (Figure 5F). It also did not affect deformability (no effect on $\mathrm{El}_{\max }$ or $\mathrm{O}_{\text {hyper }}$ ) (Figure 5G, H). However, the cells did survive for a longer time in the circulation despite an increase in osmotic fragility (Figure 5I).

\section{Discussion}

This comprehensive study in a well-characterized cohort of patients offers insight into the variable phenotypic manifestations of HS, possible causes of clinical heterogeneity and severity, and the impact of splenectomy. We show here that strong markers of moderate/severe expression of HS are: (i) lower RBC density, reflected by differences in MCHC and fractionation of $\mathrm{RBC}$ on the Percoll density gradient; (ii) reduced RBC deformability
A

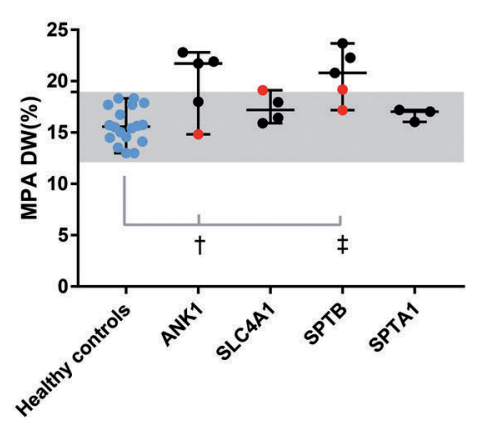

D

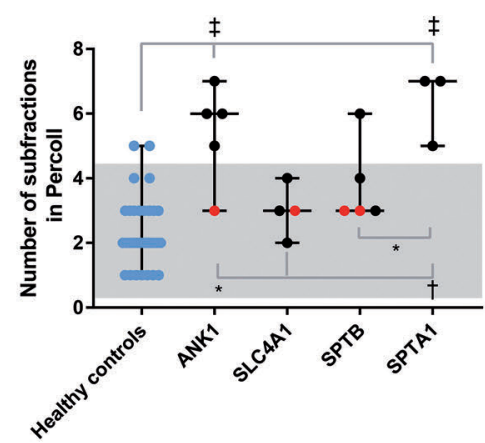

B

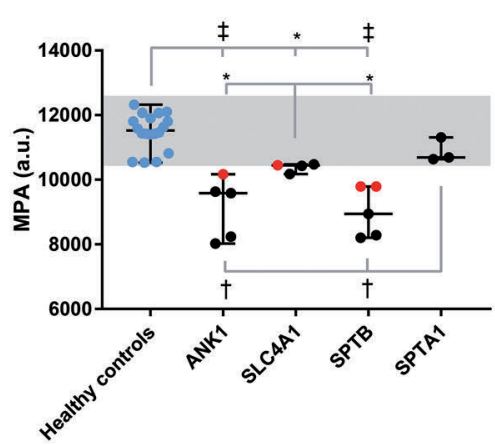

$\mathrm{E}$

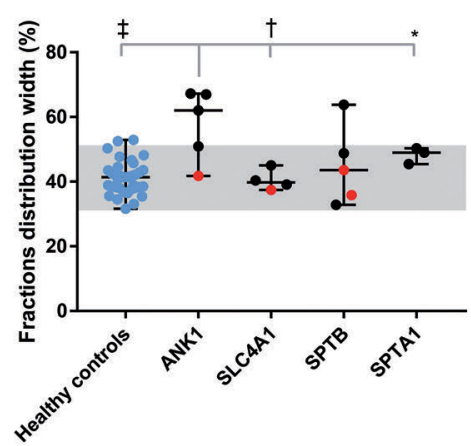

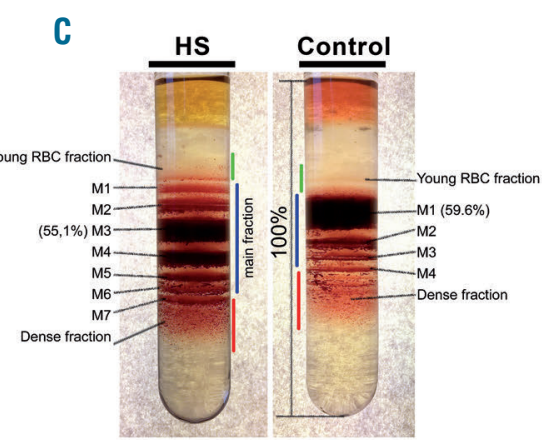

$\mathrm{F}$

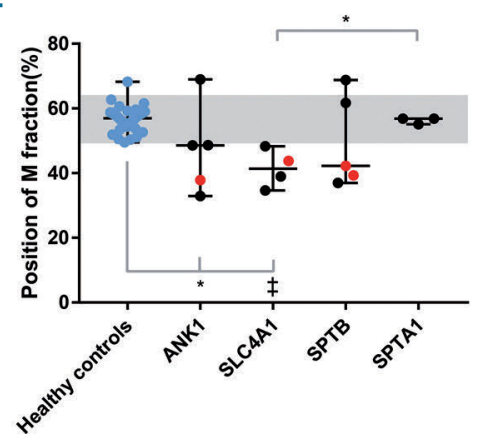

Healthy controls $\bullet$ Patients $\bullet$ Splenectomized patients

Figure 2. Red blood cell heterogeneity in hereditary spherocytosis. Red blood cell (RBC) heterogeneity was measured in healthy controls and patients with hereditary spherocytosis (HS) by microscopy (A, B) and Percoll density gradients (C-F). HS patients were grouped according to their affected genes [ANK1 (ankyrin), SLC4A1 (band 3), SPTB ( $\beta$-spectrin) and SPTA1 ( $\alpha$-spectrin)]. (A) Mean projected area distribution width (MPA DW). (B) Mean projected area (MPA). (C) An example of a blood sample from a patient with HS and a healthy control. On the samples the young RBC fraction, the main RBC fraction (M fraction) and dense RBC fractions are designated with green, blue and red lines, respectively. The $\mathrm{M}$ fraction is subdivided into subfractions (M1, M2, etc.). The position of the M fraction is calculated from the position of the most intense ( $n$ arbitrary units, a.u.) subfraction relative to the total length of the Percoll column. The HS patient has seven RBC subfractions, and the position of the most intense subfraction (i.e. subfraction M3) is lower than in the control subject (i.e. subfraction M1). (D) The number of subfractions in the RBC density gradient. (E) Fraction distribution width (\%). (F) Position of the M fraction (\%). Significant differences are noted: ${ }^{*} P \leq 0.05,{ }^{\dagger} P \leq 0.01$ or ${ }^{\ddagger} P \leq 0.001$. 
and increased membrane loss, as determined by RBC vesicle numbers and a decrease in MPA; and (iii) heterogeneity in the RBC population reflected by differences in RDW and fractionation of RBC using a Percoll gradient.

We conclude that patients with moderate/severe HS have short-lived RBC of lower density and abnormally high intercellular heterogeneity, whereas patients with mild HS have a less pronounced reduction in RBC deformability resulting in the cells living longer and being subject to progressive loss of membrane. RBC from patients with mild HS thus become denser before they are taken up by the spleen.

\section{Genotype to phenotype correlations in hereditary spherocytosis}

While previous studies were limited to protein analysis by SDS-PAGE, ${ }^{12}$ we used next-generation sequencing to establish the cause of HS. This enabled us to define the primary genetic defect unequivocally, in contrast to other conventional techniques such as SDS-PAGE, which may lead to confounding results as it may be influenced by secondary protein defects in HS. Regardless of the underlying mutation, all patients shared common features such as increases in reticulocyte counts and MCHC, dehydration and increases of RBC density and heterogeneity and an overall reduction in deformability of the RBC due to destabilization of cytoskeletal structures., ${ }^{1,29} \mathrm{We}$ also observed that red cell size, intracellular $\mathrm{K}^{+}$content and reticulocyte counts did not differ between patients with SLC4A1, ANK1, SPTB and SPTA1 mutations (Figures $1 \mathrm{H}$ and $2 \mathrm{~B}$, Table 1$)$. Within one group of patients with the same mutated protein we noted marked differences in severity and clinical manifestations of the disease, with the ANK1 group showing the greatest diversity. We also noted that patients with SPTA1 mutations had a less severe phenotype (based on hemoglobin level, reticulocyte count, RDW, intracellular $\mathrm{K}^{+}$, and EMA staining) compared to the other patients. This is in contrast with the more severe disease phenotype of patients with SPTA1 mutations reported in other studies. ${ }^{30}$ Similarly, our patients with $S P T B$ mutations presented with normal MCHC values, whereas in other studies MCHC was shown to be elevated in patients carrying SPTB mutations. ${ }^{31}$ Given the relatively small numbers of patients within each group we cannot draw firm conclusions on links between genotype and phenotypic expression.

\section{Red blood cell density as a marker of clinical severity}

Decreased hemoglobin, hematocrit and RBC counts associated with increased markers of hemolysis and erythropoietic activity were previously reported as markers of HS severity. Among the molecular mechanisms defining severity of $\mathrm{HS}$, a major role was assigned to $\mathrm{RBC}$ membrane instability and loss of membrane proteins ${ }^{28}$ (MCHC, EMA test, SDS-PAGE), along with decreased deformability as measured by osmotic gradient ektacytometry. ${ }^{5}$ In our cohort, increased disease severity was associated with membrane instability and extensive membrane and band 3 protein loss over a shorter time (Figure $4 \mathrm{~B}, \mathrm{G}-\mathrm{I})$. RBC turnover in patients with severe HS was reflected by a decrease in HbA1c levels, which were higher than those in patients with mild HS (Figure 4A). The RBC in patients with moderate/severe HS had lower $\mathrm{MCHC}$ than those of patients with mild HS (Figures $3 \mathrm{~A}$ and 4D). The average $M$ fraction density of $R B C$ from patients with moderate/severe HS did not differ from that of cells of healthy controls, whereas the cells from patients with mild HS showed an increase in density (Figure 4E)
A

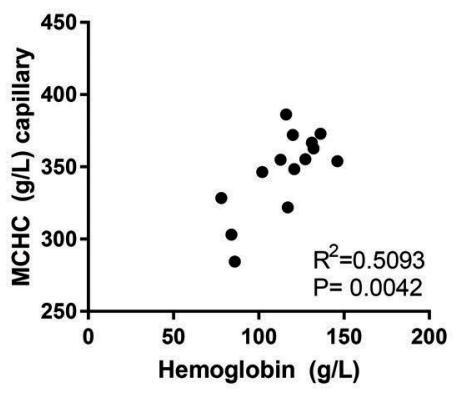

D

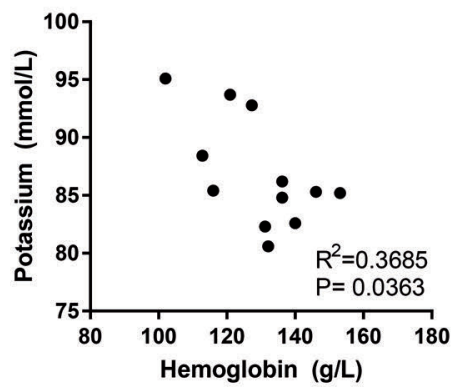

B

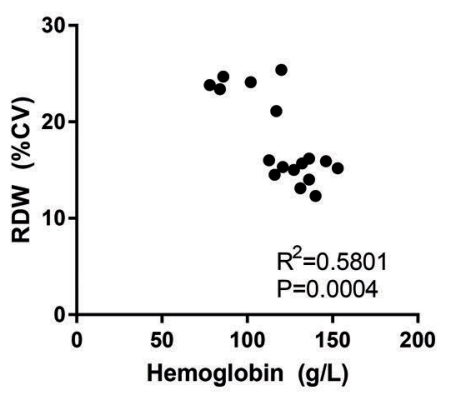

C

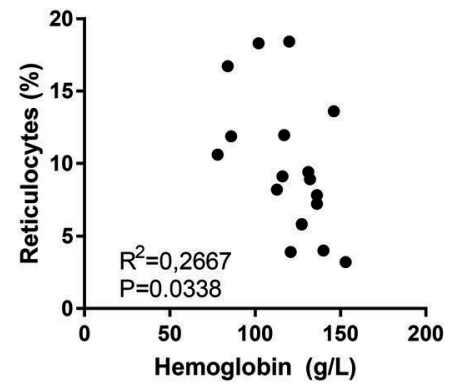

Patients

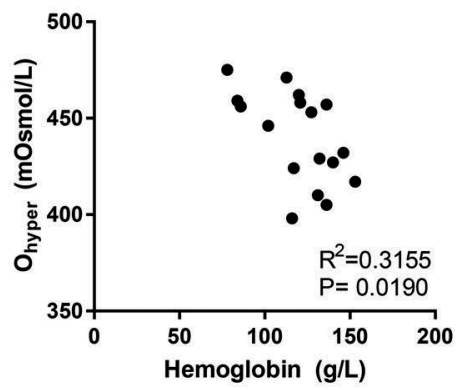

Figure 3. Red blood cell parameters and their relationship to clinical severity in unsplenectomized hereditary spherocytosis patients. (A) Mean corpuscular hemoglobin concentration ( $\mathrm{MCHC})(\mathrm{g} / \mathrm{L})$, (B) red blood cell distribution width (RDW) (percent coefficient of variation, \%CV), (C) reticulocytes (\%), (D) intracellular potassium $(\mathrm{mmol} / \mathrm{L}),(\mathrm{E})$ hypertonic osmolarity at $50 \%$ of maximal elongation $\left(\mathrm{O}_{\text {hyper }}\right)(\mathrm{mOsmol} / \mathrm{L})$. 
and lower deformability as they "aged", spending more time in the circulation getting more "senescent" than the highly unstable cells of patients with severe HS. Thus, $\mathrm{RBC}$ lifespan and decrease in $\mathrm{El}_{\max }$ appeared to be reliable markers of disease severity (Figure 4B), regardless of the genotype.

Delayed clearance of RBC in patients with mild HS allows a more gradual loss of the cell membrane (Figure 4G, Online Supplementary Figure S3), which results in bet- ter conservation of RBC deformability (Figure 4B) and the ability to form dense cells (Figure 4C-E, Online Supplementary Figure S2). Direct measurements of membrane shedding by monitoring plasma-borne vesicles is challenging due to their fast sequestration and clearance. ${ }^{32-34}$ However, higher levels of circulating RBC vesicles were detected in plasma from patients with moderate/severe HS (Figure 4G). This finding is in line with an increase in other markers of membrane loss, such as
A
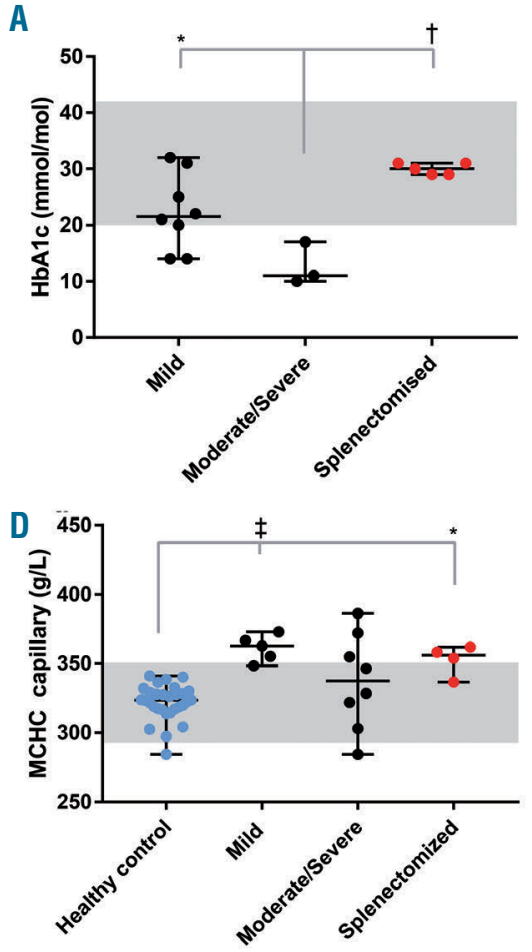

G

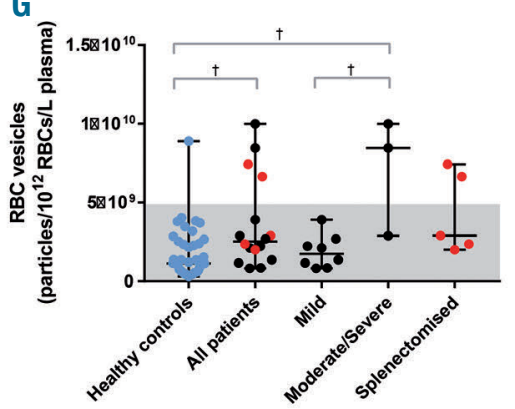

$\mathrm{J}$

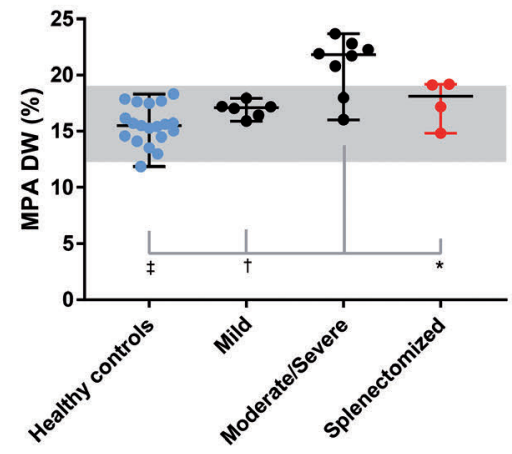

B
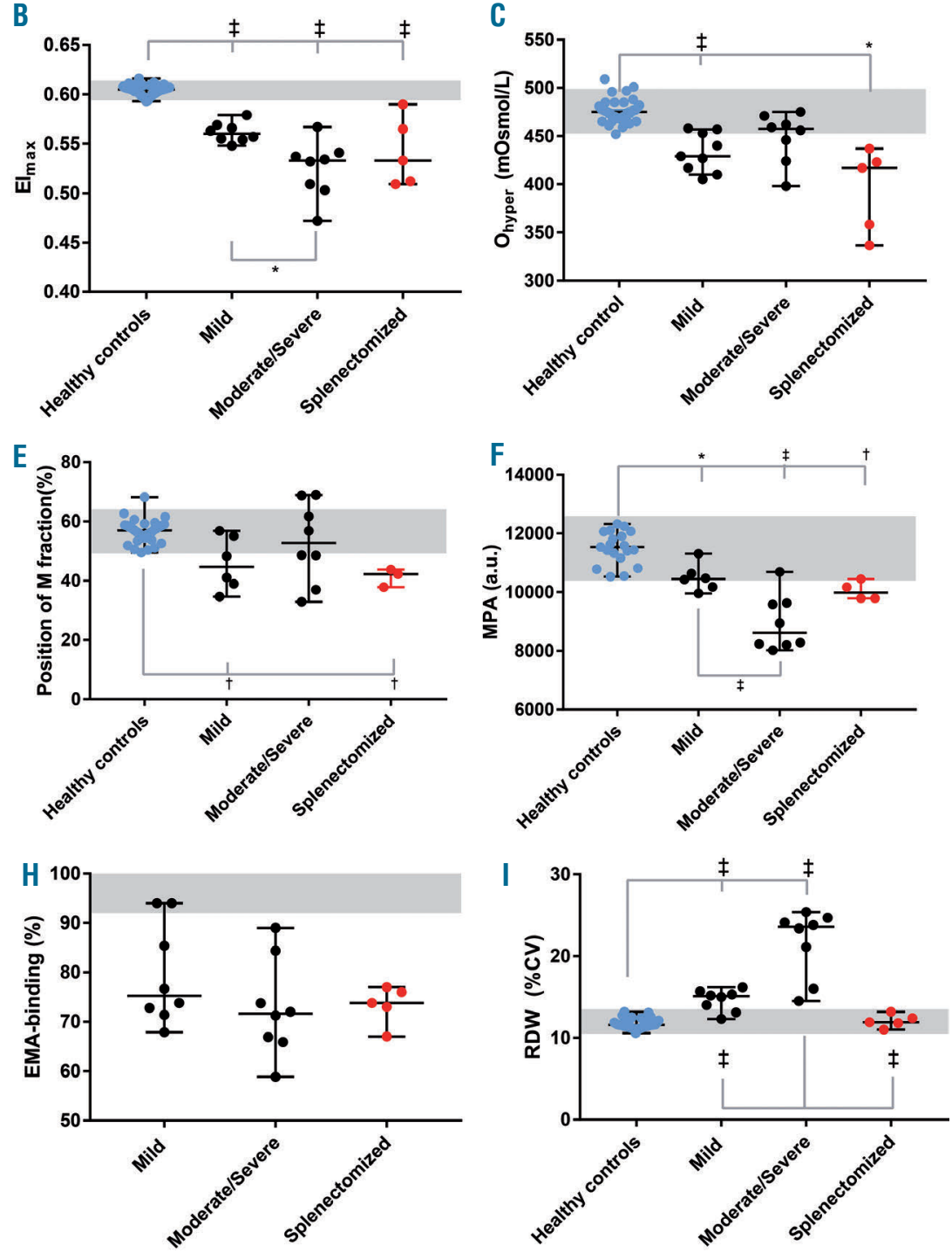

Healthy controls • Patients • Splenectomized patients

Figure 4. Red blood cell density and stability as markers of severity in hereditary spherocytosis. Patients with hereditary spherocytosis (HS) are grouped according to clinical severity (i.e. mild, moderate/severe and splenectomized) (reference range in gray area). (A) Glycated hemoglobin ( $\mathrm{HbA1c})(\mathrm{mmol} / \mathrm{mol}),(B)$ maximum deformability $\left(\mathrm{El}_{\max }\right),(\mathrm{C})$ hypertonic osmolarity at $50 \%$ of maximal elongation $\left(\mathrm{O}_{\text {hyper }}\right)(\mathrm{mOsmol} / \mathrm{L}),(\mathrm{D})$ mean corpuscular hemoglobin concentration $(\mathrm{MCHC})(\mathrm{g} / \mathrm{L})$, (E) Position of M-fraction (\%), ( $\mathrm{F}$ ) mean cell projected area (MPA) (in arbitrary units, a.u.), (G) red blood cell (RBC) vesicles (particles $/ 10^{12} \mathrm{RBC} / \mathrm{L}$ plasma), (H) eosin-5'-maleimide (EMA)-binding (\%), (I) RBC distribution width (RDW) (percent coefficient of variation, $\% \mathrm{CV}),(J)$ mean cell projected area distribution width (MPA DW). Significant differences are represented by horizontal bars, and significance levels are noted: ${ }^{*} P \leq 0.05,{ }^{\dagger} P \leq 0.01$ or ${ }^{\ddagger} P \leq 0.001$. 
changes in gross morphology toward a spherocytic form (Online Supplementary Figure S3), as well as a higher number of microcytes, as determined by quantitative digital microscopy (Online Supplementary Figure S4).

\section{Red blood cell heterogeneity and deformability} as a marker of clinical severity

Another parameter we found to reflect disease severity in $\mathrm{HS}$ is $\mathrm{RBC}$ heterogeneity. Increases in RDW, $\mathrm{RDW} /$ hemoglobin and MCHC/RDW ratios were suggested to be markers of the clinical severity of $\mathrm{HS}^{35} \mathrm{We}$ also found in our study that RDW correlates with HS severity
(Figure $3 \mathrm{~B}$ ). However, since absolute RDW values are known to vary between laboratories and depend on the age and physical activity of the subjects, ${ }^{35}$ we also used microscopy, confirming that patients with severe HS did indeed have a broader range of RBC shapes and MPA DW (Figure 4J, Online Supplementary Figure S3).

\section{Word of caution regarding automated detection of} hematocrit and mean corpuscular hemoglobin concentration in patients with hereditary spherocytosis

$\mathrm{MCHC}$ was previously suggested to be prognostic for the severity of HS in non-splenectomized patients. ${ }^{9,11} \mathrm{We}$
A

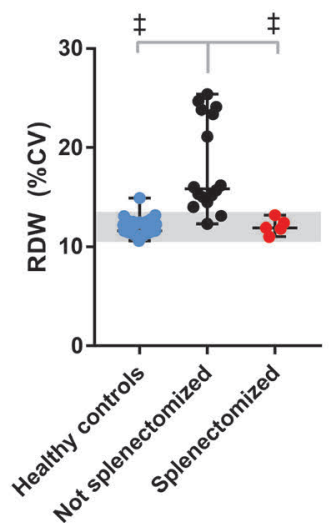

B

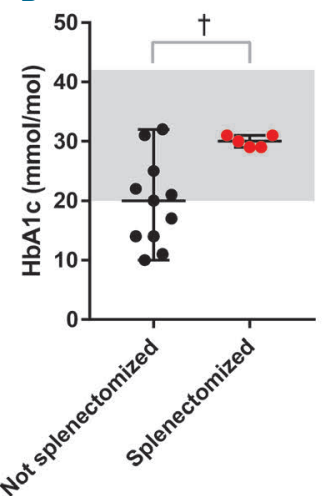

C

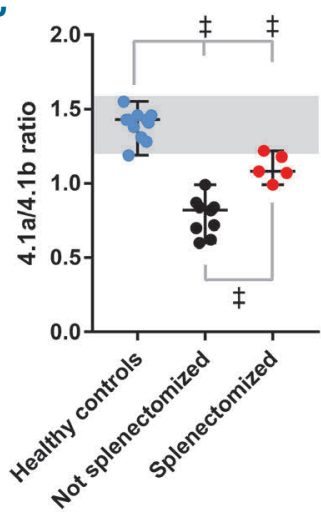

D

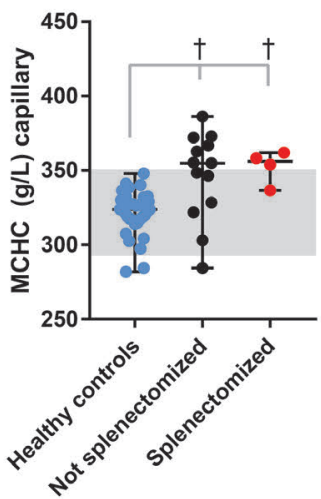

E

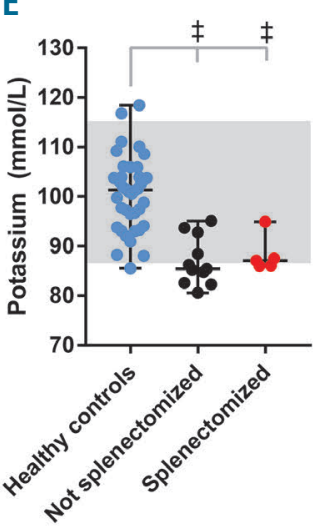

$\mathrm{F}$

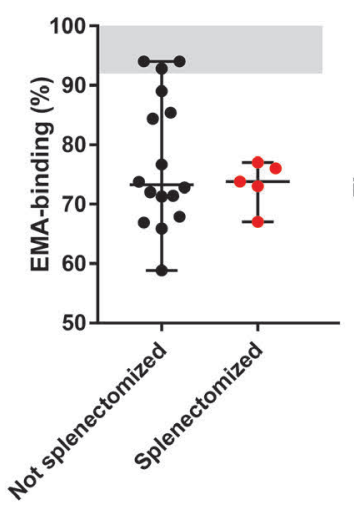

G

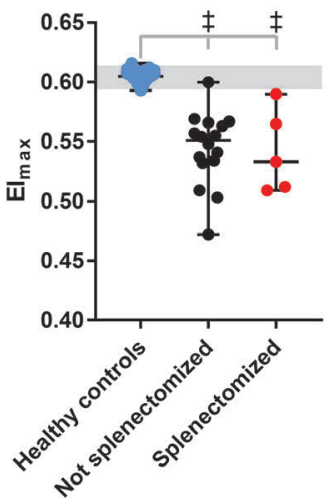

H

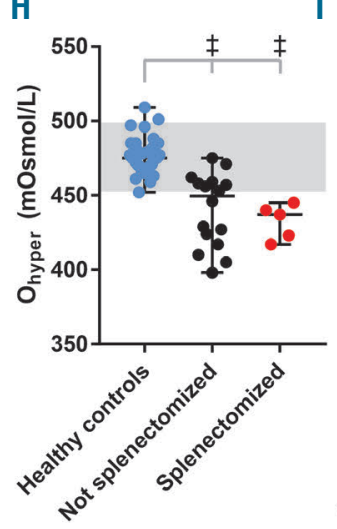

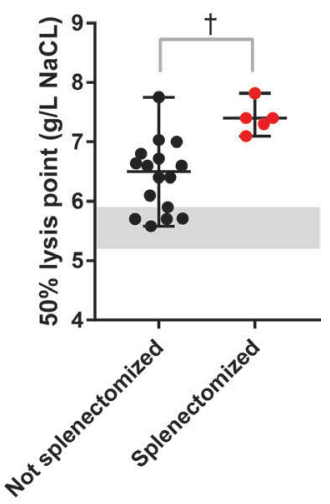

Healthy controls •Patients • Splenectomized patients

Figure 5. Role of splenectomy on red blood cell parameters in hereditary spherocytosis. Blue circles represent healthy controls, black circles represent (unsplenectomized) hereditary spherocytosis (HS) patients and red circles represent splenectomized HS patients. HS patients are grouped according to clinical severity (i.e. mild, moderate/severe and splenectomized). (A) Red cell distribution width (RDW (percent coefficient of variation, \%CV), (B) glycated hemoglobin (HbA1c) ( $\mathrm{mmol} / \mathrm{mol}),(\mathrm{C})$ band 4.1a/4.1b ratio, (D) mean corpuscular hemoglobin concentration (MCHC) (g/L), (E) intracellular potassium (mmol/L), (F) eosin-5'-maleimide (EMA)-binding hemoglobin (\%), (G) maximum deformability $\left(\mathrm{EI}_{\max }\right),(\mathrm{H})$ hydration state of the red blood cells reflected by hypertonic osmolarity at $50 \%$ of maximal elongation $\left(\mathrm{O}_{\text {hyper }}\right)(\mathrm{mOsmol} / \mathrm{L})$, (I) $50 \%$ lysis point in the osmotic fragility test $(\mathrm{g} / \mathrm{L} \mathrm{NaCl})$. Significant differences are noted: ${ }^{*} P \leq 0.05,{ }^{\dagger} P \leq 0.01$ or ${ }^{\ddagger} P \leq 0.001$.

Table 2. Summary of parameters that characterize disease severity in hereditary spherocytosis.

\begin{tabular}{|c|c|c|c|c|c|c|}
\hline Disease Severity & МСНC & $\begin{array}{c}\text { Density } \\
\text { (Percoll, } \mathbf{0}_{\text {bpee }} \text { ) }\end{array}$ & RDW & Reticulocytes & $\begin{array}{c}\text { Deformability } \\
\left(\mathrm{E}_{\mathrm{max}}\right)\end{array}$ & $\begin{array}{l}\text { Membrane loss } \\
\text { (vesiculation) }\end{array}$ \\
\hline Mild & $\uparrow \uparrow$ & $\uparrow \uparrow$ & $=$ & $\uparrow$ & $\downarrow$ & $\uparrow$ \\
\hline Moderate/severe & $\uparrow$ & $\uparrow$ & $\uparrow \uparrow$ & $\uparrow \uparrow$ & $\downarrow \downarrow$ & $\uparrow \uparrow$ \\
\hline Splenectomized & $\uparrow \uparrow$ & $=$ & $=$ & $\uparrow$ & $=$ & $=$ \\
\hline
\end{tabular}

The $\uparrow$ symbol indicates increased compared to healthy controls, $\downarrow$ indicates decreased compared to healthy controls and = indicates the same as in healthy controls. MCHC: mean corpuscular hemoglobin concentration; RDW: red cell distribution width; $\mathrm{EI}_{\max }$ : maximal elongation index, 
used a capillary-based method with determination of the hematocrit to measure $\mathrm{MCHC}$. In line with previous observations, ${ }^{36-38}$ we found that automated measurements of $\mathrm{MCHC}$ of pathological RBC are imprecise for HS patients (see Online Supplementary Figure S5). Inaccuracy of automated $\mathrm{MCHC}$ detection of dehydrated RBC has been discussed for over 30 years. $^{36}$ It results from a substantial overestimation of $\mathrm{MCV}$, both values being reliant on hematocrit detection or calculation methods. ${ }^{39,40}$ As mentioned previously, others have shown that increased hemoglobin concentrations correlate with milder disease severity scores in HS. ${ }^{9,11}$ We show that MCHC, calculated based on the capillary hematocrit, correlated with blood hemoglobin content (Figures 1G, 3A, and 4D).

\section{Conclusions}

This study reveals the factors defining RBC longevity and erythropoietic activity in patients with HS. These factors include membrane stability, which in turn depends on the localization of mutations affecting vertical or horizontal interactions within the membrane cytoskeleton and on the presence of splenic filtering capacity. Mild HS is asso- ciated with prolonged survival of RBC in the circulation, allowing greater loss of membrane, which results in smaller and denser RBC. Shorter-lived, unstable RBC from patients with severe HS phenotype are more heterogeneous and less dense, as reflected by lower MCHC.

Parameters that specifically mark clinical severity in HS are summarized in Table 2 and are RBC density (MCHC, Percoll, $\left.\mathrm{O}_{\text {hyper }}\right), \mathrm{RBC}$ deformability $\left(\mathrm{EI}_{\max }\right)$, and $\mathrm{RBC}$ heterogeneity (RDW). These parameters may be used to monitor the success of supportive therapy and assist in the development of new personalized treatment regimens.

\section{Funding}

Funding for the research leading to these results was received from the European Seventh Framework Program under grant agreement number 602121 (CoMMiTMenT) and from the European Union's Horizon 2020 Research and Innovation Programme under grant agreement number 675115 - RELEVANCE - H2020-MSCA-ITN-2015/H2020-MSCA-ITN2015. This work was generated within the European Reference Network on Rare Hematological Diseases (ERNEuroBloodNet) - FPA No. 739541

\section{References}

1. Perrotta S, Gallagher PG, Mohandas N. Hereditary spherocytosis. Lancet. 2008;372 (9647):1411-1426.

2. Morton NE, Mackinney a a, Kosower N, Schilling RF, Gray MP. Genetics of spherocytosis. Am J Hum Genet. 1962;170-184.

3. Gallagher PG. Hereditary elliptocytosis: spectrin and protein 4.1R. Semin Hematol. 2004;41(2):142-164

4. Da Costa L, Mohandas N, Sorette M, Grange MJ, Tchernia G, Cynober T. Temporal differences in membrane loss lead to distinct reticulocyte features in hereditary spherocytosis and in immune hemolytic anemia. Blood. 2001;98(10):2894-2899.

5. Lazarova E, Gulbis B, Oirschot B van, van Wijk R. Next-generation osmotic gradient ektacytometry for the diagnosis of hereditary spherocytosis: interlaboratory method validation and experience. Clin Chem Lab Med. 2017;55(3):394-402

6. Huisjes R, Bogdanova A, van Solinge WW, Schiffelers RM, Kaestner L, van Wiik R. Squeezing for life - properties of red blood cell deformability. Front Physiol. 2018;9:656.

7. Michaels LA, Cohen AR, Zhao H, Raphael RI, Manno CS. Screening for hereditary spherocytosis by use of automated erythrocyte indexes. J Pediatr. 1997;130(6):957-960.

8. Cynober T, Mohandas N, Tchernia G. Red cell abnormalities in hereditary spherocytosis: Relevance to diagnosis and understanding of the variable expression of clinical severity. J Lab Clin Med. 1996;128(3):259-269.

9. Rocha S, Costa E, Rocha-Pereira P, et al. Complementary markers for the clinical severity classification of hereditary spherocytosis in unsplenectomized patients. Blood Cells Mol Dis. 2011;46(2):166-170.

10. Eber SW, Armbrust R, Schröter W. Variable clinical severity of hereditary spherocytosis: relation to erythrocytic spectrin concentration, osmotic fragility, and autohemolysis. J Pediatr. 1990;117 (3):409-416.
11. Bolton-Maggs PHB, Langer JC, Iolascon A Tittensor P, King M-J, General Haematology Task Force of the British Committee for Standards in Haematology. Guidelines for the diagnosis and management of hereditary spherocytosis - 2011 update. Br J Haematol. 2012;156(1):37-49.

12. King MJ, Behrens J, Rogers C, Flynn C, Greenwood D, Chambers K. Rapid flow cytometric test for the diagnosis of membrane cytoskeleton-associated haemolytic anaemia. Br J Haematol. 2000;111 (3):924 933.

13. Bosch FH, Werre JM, Schipper L, et al. Determinants of red blood cell deformability in relation to cell age. Eur J Haematol. 1994;52(1):35-41.

14. Waugh RE, Narla M, Jackson CW, Mueller TJ, Suzuki T, Dale GL. Rheologic properties of senescent erythrocytes: loss of surface area and volume with red blood cell age. Blood. 1992;79(5):1351-1358

15. Bosman GJCGM. Survival of red blood cells after transfusion: processes and consequences. Front Physiol. 2013;4:376.

16. Vives Corrons JL, Besson I. Red cell membrane $\mathrm{Na}+$ transport systems in hereditary spherocytosis: relevance to understanding the increased $\mathrm{Na}+$ permeability. Ann Hematol. 2001;80(9):535-539.

17. Gallagher PG. Disorders of erythrocyte hydration. Blood. 2017;130(25):2699-2708.

18. De Franceschi L, Olivieri $O$, Miraglia del Giudice E, et al. Membrane cation and anion transport activities in erythrocytes of hereditary spherocytosis: effects of different membrane protein defects. Am J Hematol. 1997;55(3):121-128.

19. Makhro A, Huisjes R, Verhagen LP, et al. Red cell properties after different modes of blood transportation. Front Physiol. 2016;7:288.

20. Jokinen $\mathrm{CH}$, Swaim WR, Nuttall FQ. A case of hereditary xerocytosis diagnosed as a result of suspected hypoglycemia and observed low glycohemoglobin. J Lab Clin Med. 2004;144(1):27-30.

21. Da Costa L, Suner L, Galimand I, et al.
Diagnostic tool for red blood cell membrane disorders: assessment of a new generation ektacytometer. Blood Cells Mol Dis. 2016;56(1):9-22

22. Parpart AK, Lorenz PB, Parpart ER, Gregg JR, Chase AM. The osmotic resistance (fragility) of human red cells. J Clin Invest. 1947;26(4): 636-640.

23. King $\mathrm{M}-\mathrm{J}$, Telfer $\mathrm{P}$, MacKinnon $\mathrm{H}$, et al. Using the eosin-5-maleimide binding test in the differential diagnosis of hereditary spherocytosis and hereditary pyropoikilocytosis Cytometry B Clin Cytom. 2008;74(4):244250

24. Ghosh S, Chakraborty I, Chakraborty M Mukhopadhyay A, Mishra R, Sarkar D. Evaluating the morphology of erythrocyte population: an approach based on atomic force microscopy and flow cytometry. Biochim Biophys Acta. 2016;1858(4):671681.

25. Lutz HU, Bogdanova A. Mechanisms tag ging senescent red blood cells for clearance in healthy humans. Front Physiol. 2013; 4:387.

26. Makhro A, Kaestner L, Bogdanova A. NMDA receptor activity in circulating red blood cells: methods of detection. Methods Mol Biol. 2017;1677:265-282.

27. Huisjes $R$, van Solinge WW, Levin MD, van Wijk R, Riedl JA. Digital microscopy as a screening tool for the diagnosis of hereditary hemolytic anemia. Int J Lab Hematol. 2018;40(2):159-168.

28. van Vuren A, van der Zwaag B, Huisjes R et al. The Complexity of Genotype-Phenotype Correlations in Hereditary Spherocytosis: A Cohort of 95 Patients. Hemasphere. 2019 Aug 7;3(4):e276.

29. Narla J, Mohandas N. Red cell membrane disorders. Int J Lab Hematol. 2017;39 Supp 1:47-52

30. Chonat S, Risinger M, Dagaonkar N, et al The spectrum of alpha-spectrin associated hereditary spherocytosis. Blood. 2015;126 (23):941.

31. Maciag M, Płochocka D, Adamowicz 
Salach A, Burzyńska B. Novel beta-spectrin mutations in hereditary spherocytosis associated with decreased levels of mRNA. Br J Haematol. 2009;146(3):326-332.

32. Willekens FLA, Werre JM, Groenen-Döpp YAM, Roerdinkholder-Stoelwinder B, de Pauw B, Bosman GJCGM. Erythrocyte vesiculation: a self-protective mechanism? Br J Haematol. 2008;141 (4):549-556.

33. Ciana A, Achilli C, Gaur A, Minetti G. Membrane remodelling and vesicle formation during ageing of human red blood Cells. Cell Physiol Biochem. 2017;42(3):11271138.

34. Willekens FLA, Werre JM, Kruiit JK, et al. Liver Kupffer cells rapidly remove red blood cell-derived vesicles from the circulation by scavenger receptors. Blood. 2005;105(5): 2141-2145.

35. Salvagno GL, Sanchis-Gomar F, Picanza A, Lippi G. Red blood cell distribution width: a simple parameter with multiple clinical applications. Crit Rev Clin Lab Sci. 2015; 52(2):86-105.

36. Mohandas N, Clark MR, Kissinger S, Bayer C, Shohet SB. Inaccuracies associated with the automated measurement of mean cell hemoglobin concentration in dehydrated cells. Blood. 1980;56(1):125-128.

37. Berda-Haddad Y, Faure C, Boubaya M, et al. Increased mean corpuscular haemoglobin concentration: artefact or pathological con- dition? Int J Lab Hematol. 2017;39(1):32-41. 38. Zandecki M, Genevieve F, Gerard J, Godon A. Spurious counts and spurious results on haematology analysers: a review. Part II white blood cells, red blood cells, haemoglobin, red cell indices and reticulocytes. Clin Lab Haematol. 2007;29(1):21-41.

39. Kokholm G. Simultaneous measurements of blood $\mathrm{pH}, \mathrm{pCO} 2, \mathrm{pO} 2$ and concentrations of hemoglobin and its derivates-a multicenter study. Scand J Clin Lab Invest. 1990;50 (sup203):75-86.

40. O'Connor G, Molloy AM, Daly L, Scott JM. Deriving a useful packed cell volume estimate from haemoglobin analysis. J Clin Pathol. 1994;47(1):78-79. 